\title{
Alumina Ceramic Based High-Temperature Performance of Wireless Passive Pressure Sensor
}

\author{
Bo WANG ${ }^{1}$, Guozhu $\mathrm{WU}^{2,3^{*}}$, Tao GUO ${ }^{2,3}$, and Qiulin TAN ${ }^{2,3}$ \\ ${ }^{1}$ School of Computer Science and Control Engineering, North University of China, Taiyuan, 030051, China \\ ${ }^{2}$ Key Laboratory of Instrumentation Science \& Dynamic Measurement, Ministry of Education, North University of China, \\ Taiyuan, 030051, China \\ ${ }^{3}$ Science and Technology on Electronic Test and Measurement Laboratory, North University of China, Taiyuan, 030051, \\ China \\ ${ }^{*}$ Corresponding author: Guozhu WU \\ E-mail: pillar921@163.com
}

\begin{abstract}
A wireless passive pressure sensor equivalent to inductive-capacitive (LC) resonance circuit and based on alumina ceramic is fabricated by using high temperature sintering ceramic and post-fire metallization processes. Cylindrical copper spiral reader antenna and insulation layer are designed to realize the wireless measurement for the sensor in high temperature environment. The high temperature performance of the sensor is analyzed and discussed by studying the phase-frequency and amplitude-frequency characteristics of reader antenna. The average frequency change of sensor is $0.68 \mathrm{kHz} /{ }^{\circ} \mathrm{C}$ when the temperature changes from $27^{\circ} \mathrm{C}$ to $700{ }^{\circ} \mathrm{C}$ and the relative change of twice measurements is $2.12 \%$, with high characteristic of repeatability. The study of temperature-drift characteristic of pressure sensor in high temperature environment lays a good basis for the temperature compensation methods and insures the pressure signal readout accurately.
\end{abstract}

Keywords: Pressure sensor; wireless passive; high temperature; zero drift; resonant frequency

Citation: Bo WANG, Guozhu WU, Tao GUO, and Qiulin TAN, "Alumina Ceramic Based High-Temperature Performance of Wireless Passive Pressure Sensor,” Photonic Sensors, 2016, 6(4): 328-332.

\section{Introduction}

Pressure signal acquisition has been greatly demanded in harsh environment, featuring turbine engine design, and petroleum exploration, whereas all of them require the sensor capable of operating at more than $200{ }^{\circ} \mathrm{C}$ [1-3]. Existing sensors are able to meet the requirements in the measuring range, frequency response, and accuracy, while they can only play roles in some of "moderate" environments $[4,5]$. For now, the pressure sensor application is still deficient in at least the following two aspects, or just meets the minimum demands: one is ultra-high temperature environment, the other is metallic environment, which causes serious electromagnetic interference. Currently, pressure sensors applied for high temperature have been carried out in-depth research, including silicon on insulator (SOI) high temperature pressure sensor [6, 7], $\mathrm{SiC}$ high temperature pressure sensor [8], silicon-sapphire pressure sensor [9], fiber optic pressure sensor [10], [11], surface acoustic wave (SAW) pressure sensor $[12,13]$, and the pressure sensor equivalent to inductive-capacitive (LC) resonance circuit based pressure sensor [14-16].

A pressure sensor based on $\mathrm{LC}$ resonance circuit

Received: 11 January 2016 / Revised: 2 June 2016

(C) The Author(s) 2016. This article is published with open access at Springerlink.com

DOI: $10.1007 / \mathrm{s} 13320-016-0313-0$

Article type: Regular 
uses alumina ceramic as the sensor substrate, which can survive in high temperature above $1000^{\circ} \mathrm{C}$. The sensor is under test at different temperatures to analyze and discuss the impact on the pressure signal readout, which provides valuable reference to the research of temperature compensation.

\section{Working principle}

The pressure sensor can be considered as an LC resonance circuit, which consists of inductance connected in series with capacitance and resistance. $L_{s}$ is inductance coil, $R_{S}$ is equivalent resistance, and $C_{s}$ is variable capacitance that responses to outer pressure. The resonance frequency of sensor $f_{0}$ and quality factor $Q$, neglecting the parasitic capacitance, can be approximately expressed as

$$
\begin{gathered}
f_{0}=\frac{1}{2 \pi \sqrt{L_{s} C_{s}}} \\
Q=\frac{1}{R} \sqrt{\frac{L_{s}}{C_{s}} .}
\end{gathered}
$$

The wireless sensing scheme is presented here by utilizing near-field magnetic coupling between the reader antenna and sensor. By extracting a certain impedance characteristic parameter (amplitude and phase), the variation of sensor frequency can be detected with high resolution. The equivalent wireless coupling circuit is shown in Fig. 1, where $L_{a}$ is the inductance of antenna coil, $R_{a}$ is the equivalent resistance of antenna, and $M$ is the coefficient of mutual inductance between reader antenna and sensor.

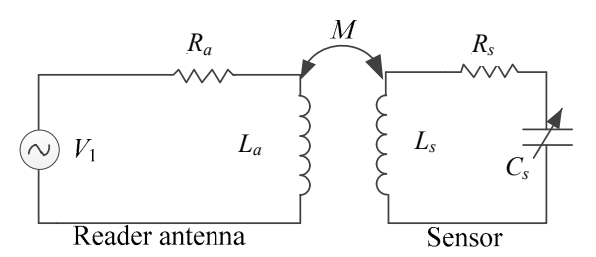

Fig. 1 Equivalent circuit of wireless coupling scheme.

\section{Design and fabrication}

The pressure sensor was fabricated separately by using high temperature ceramic sintering and post-fire metallization process. ESL 44007 green tape was chosen as the sensor substrate, and ESL 9598-G cermet silver/platinum conductor was selected as the screen printing paste. The properties of green tape composed of 99 percent alumina are listed in Table 1. The main fabrication process of the pressure sensor can be roughly divided into punching, via filling and carbon membrane filling, lamination, high-temperature sintering, screen printing, and low temperature sintering as shown in Fig. 2. A punching machine was used to drill a cavity in Layer 2 and via holes in all three layers firstly. Next, via holes were filled with metal paste to achieve electrical connection throughout the multilayer tapes. After via holes filling, Layers 2 and 3 were precisely stacked by the collating machine. Then, a carbon membrane that had the same dimensions as the cavity was placed into the cavity of the stacked two-layer substrate to support the pressure-sensitive membranes avoiding from collapsing and cracking during the lamination process. After carbon membrane filling, all three layers were stacked together to form a complete cavity. Then, the ceramic tape was placed into the lamination machine under a pressure of $21 \mathrm{MPa}$ and a temperature of $70^{\circ} \mathrm{C}$ for 20 minutes to make the multilayered tapes sufficiently bonded. Finally, the laminated substrate was sintered in a furnace at temperature of $1500^{\circ} \mathrm{C}$ for 2 hours. It should be noticed that in the high temperature sintering process, with an adequate temperature-time curve, the carbon membrane would volatilize through the apertures of the ceramic tapes and the cavity would remain intact. After sintering the alumina substrate embedded with air cavity, the inductor and capacitor patterns were printed on the surface of substrate by the screen-printing technique. Afterwards, the semi-finished sensor was placed into a furnace at $125^{\circ} \mathrm{C}$ for 15 minutes to dry the metal paste and sintered at peak temperature of $850{ }^{\circ} \mathrm{C}$ for 10 minutes. The profile of sensor structure and fabricated pressure sensor with thickness of $0.38 \mathrm{~mm}$ is shown in Fig. 3. 
Table 1 Characteristics of the ESL 44007 green tape.

\begin{tabular}{cc}
\hline Quantity & Value \\
\hline Unfired thickness & $\sim 200 \mu \mathrm{m}$ \\
Tape uniformity & $150 \pm 10 \%$ \\
$X, Y$ shrinkage & $15.8 \pm 1 \%$ \\
Relative permittivity $\left(25^{\circ} \mathrm{C}, 1 \mathrm{MHz}\right)$ & 9 \\
Dielectric loss $\left(25^{\circ} \mathrm{C}, 1 \mathrm{MHz}\right)$ & 0.0001 \\
\hline
\end{tabular}

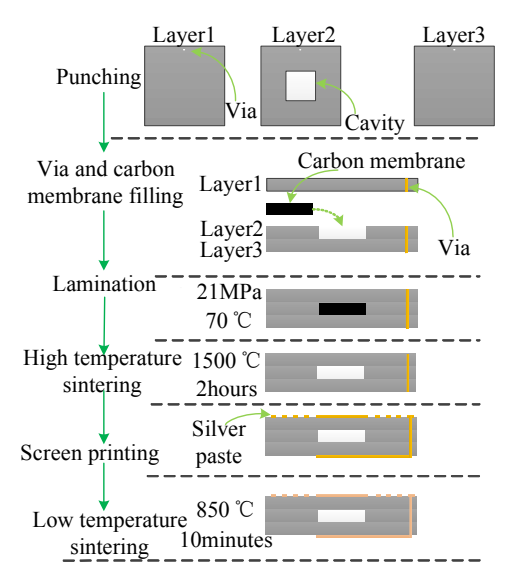

Fig. 2 Fabrication process of pressure sensor.

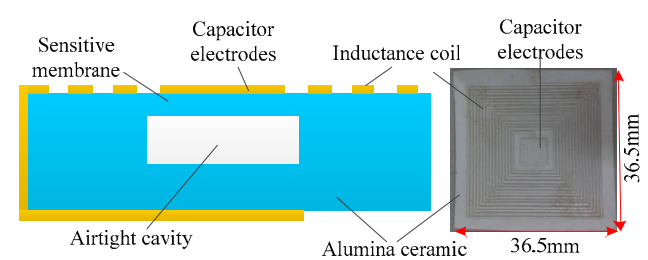

Fig. 3 Profile of pressure sensor structure and fabricated pressure sensor.

To wireless coupling with the sensor, a proper reader antenna was designed to enhance the sensing distance and signal strength of sensor. Copper was used to enwind as a cylindrical spiral antenna owing to the characteristics of low cost and excellent mechanical and electrical performance.

High temperature would change some characteristic parameters of reader antenna (inductance, resistance, etc.), thus affecting the signal readout of the sensor (signal strength and frequency deviation, etc.). To improve the accuracy of sensor signal measurement, an insulation layer was designed to insure that the reader antenna was working in a normal temperature range, and therefore, minimized the influence of reader antenna. The sensor was put in the high temperature zone while spiral antenna was placed in the room temperature zone, and two of them were separated by the insulation layer. The schematic diagram is shown in Fig. 4.

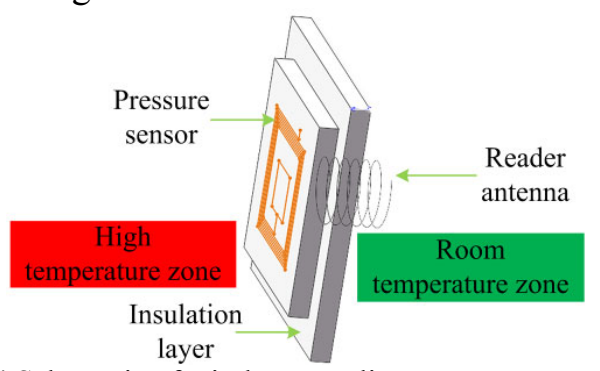

Fig. 4 Schematic of wireless coupling measurement.

\section{Measurement and discussion}

The high temperature performance of sensor measurement system is shown in Fig. 5. Muffle furnace worked as a heat source in atmospheric environment. Impedance analyzer was used to detect the phase-frequency and amplitude-frequency characteristics of reader antenna.

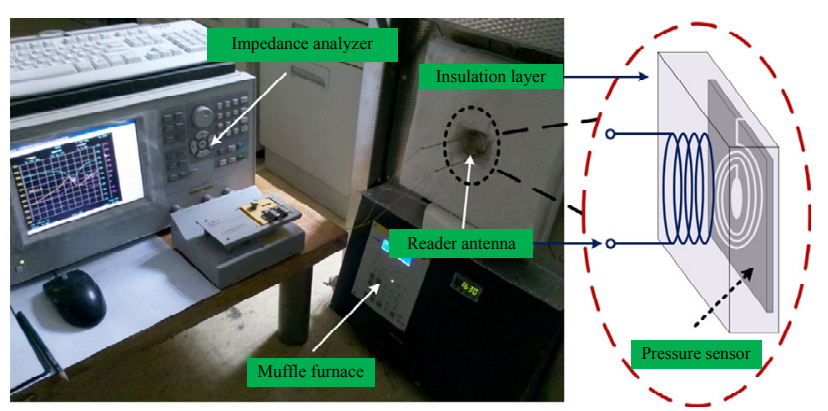

Fig. 5 High temperature measurement system of pressure sensor.

The sensor was measured at a distance of $10 \mathrm{~mm}$ (maximum measurement distance is $50 \mathrm{~mm}$ ) away from reader antenna to get a clear frequency peak signal. Figure 6 presents the temperature of the insulation layer at different sides. The temperature rises uniformly inside the muffle furnace, while the outside of furnace goes up slowly, which ensures the accuracy readout of sensor signal.

The amplitude and phase of reader antenna corresponding to frequency are presented in Figs. 7 and 8 respectively when temperature rose from $27{ }^{\circ} \mathrm{C}$ to $700{ }^{\circ} \mathrm{C}$. The resonant frequency and coupling strength of pressure sensor decreased when the temperature increased. The average change in the resonance frequency versus temperature was $0.68 \mathrm{kHz} /{ }^{\circ} \mathrm{C}$, namely zero drift $0.68 \mathrm{kHz} /{ }^{\circ} \mathrm{C}$. 


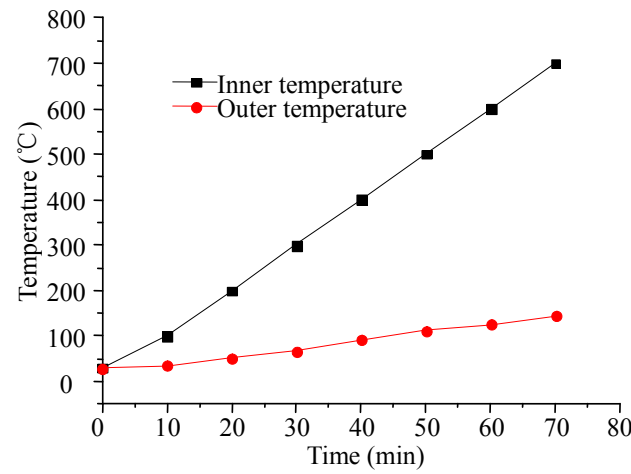

Fig. 6 Inner and outer temperature of insulation layer.

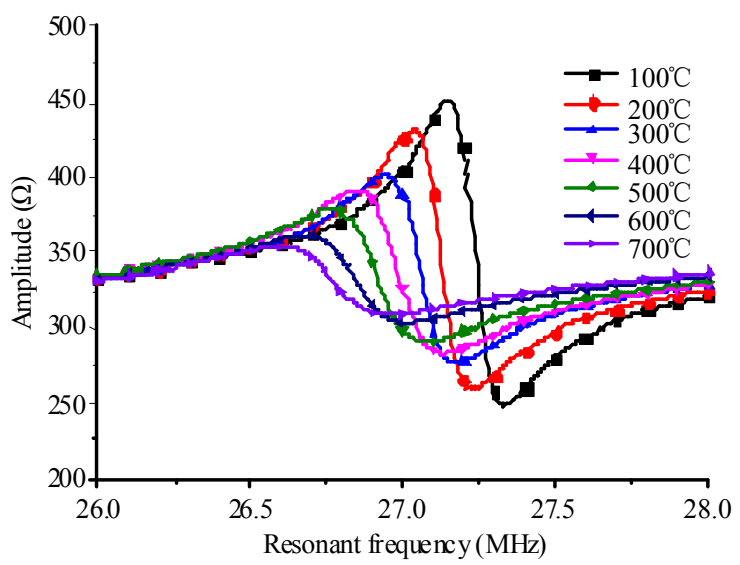

Fig. 7. Amplitude-frequency characteristic of reader antenna at different temperatures.

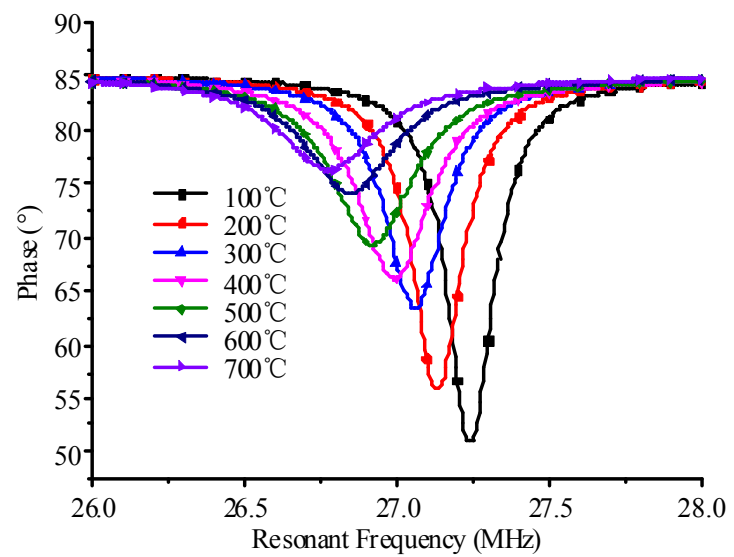

Fig. 8 Phase-frequency characteristic of reader antenna at different temperatures.

With the evaluated temperature, the alumina ceramic relative permittivity increased, thus increasing the $C_{s}$, while inductance $L_{s}$ merely changed due to the low coefficient of thermal expansion of alumina ceramic. Therefore, the resonant frequency of the sensor changed accordingly with the temperature according to (1). The inductive-capacitive resonance circuit was formed by the silver conductor. The resistivity of silver changes with temperature and can be expressed as

$$
R_{1}=R_{2} *\left[a *\left(T_{1}-T_{2}\right)\right]
$$

where $R_{1}$ is the resistivity value adjusted to $T_{1}, R_{2}$ is the resistivity value known or measured at temperature $T_{2}, a$ is the temperature coefficient, for Ag being 0.0038, $T_{1}$ is the temperature at which resistivity value needs to be known, and $T_{2}$ is the temperature at which known or measured value was obtained. The resistance of the sensor at $27^{\circ} \mathrm{C}$ measured by a multimeter is $5.7 \Omega$; by using (3), the resistance of the sensor at $700^{\circ} \mathrm{C}$ is $14.58 \Omega$. The increased resistivity causes a decrease in $Q$ value, which will make the coupling strength gradually weakened and the frequency curve unrecognizable.

When the muffle furnace cools down while other conditions remain unchanged, rise temperature to $700{ }^{\circ} \mathrm{C}$ again to proceed repetitive experiment. Figure 9 represents the extracted value of resonant frequency of sensor versus temperature. From the second measurement result, zero drifts was $0.7 \mathrm{kHz} /{ }^{\circ} \mathrm{C}$, with a good repetition compared with the first time.

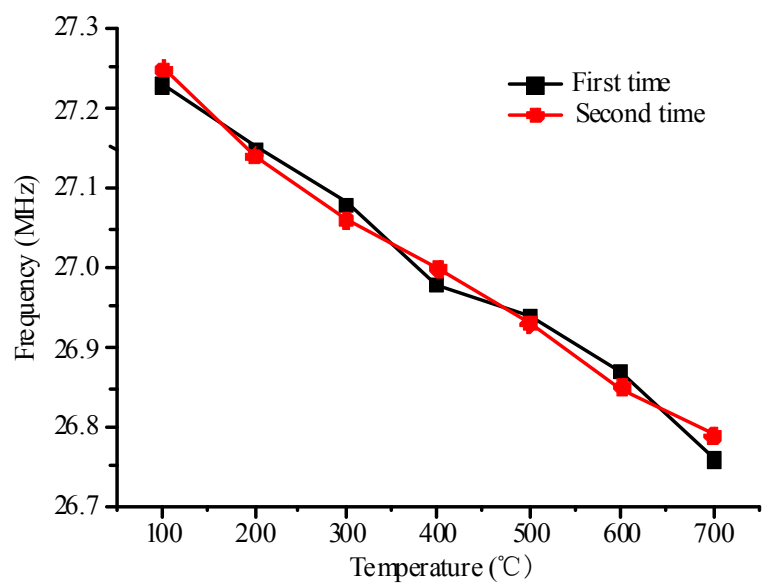

Fig. 9 extracted resonant frequency of senor versus temperature.

\section{Conclusions}

The wireless passive pressure sensor based on alumina ceramic is presented here to analyze its high temperature performance. The insulation layer was designed to ensure the stability of reader antenna in 
high temperature and improve the reliability of the performance of sensor in high temperature. When the temperature rose, the resonant frequency of sensor decreased with a trend of quadratic curve, and the coupling strength decreased gradually. Through experimental analysis, zero drift has great impact on the accuracy of sensor signal readout, and it is mainly affected by the relative permittivity of alumina ceramic and the resistivity of silver conductor. Zero-drift could be eliminated by improving the sensitivity of pressure sensor or putting forward a kind of temperature compensation algorithm to reduce the impact on the pressure signal readout.

\section{Acknowledgment}

This work was supported by the National Natural Science Foundation of China (Grant No. 61471324) and the Outstanding Young Talents Support Plan of Shanxi province.

Open Access This article is distributed under the terms of the Creative Commons Attribution 4.0 International License (http://creativecommons.org/licenses/by/4.0/), which permits unrestricted use, distribution, and reproduction in any medium, provided you give appropriate credit to the original author(s) and the source, provide a link to the Creative Commons license, and indicate if changes were made.

\section{References}

[1] W. J. Pulliam, P. M. Russler, and R. S. Fielder, "High-temperature high-bandwidth fiber optic MEMS pressure-sensor technology for turbine engine component testing," SPIE, 2002, 4578: 229-238.

[2] R. W. Johnson, J. L. Evans, P. Jacobsen, J. R. Thompson, and M. Christopher, "The changing automotive environment: High-temperature electronics," IEEE Transactions on Electronics Packaging Manufacturing, 2004, 27(3): 164-176.

[3] C. V. Netten, "Design of a small personal air monitor and its application in aircraft," Science of the Total Environment, 2009, 407(3): 1206-1210.

[4] S. E. Zhu, M. K. Ghatkesar, C. Zhang, and G. C. A. M. Janssen, "Graphene based piezoresistive pressure sensor," Applied Physics Letters, 2013, 102(16): 161904-1-161904-3.
[5] G. Chitnis, T. Maleki, B. Samuels, L. B. Cantor, and B. Ziaie, "A minimally invasive implantable wireless pressure sensor for continuous IOP monitoring," IEEE Transactions on Biomedical Engineering, 2013, 60(1): 250-256.

[6] M. Narayanaswamy, R. J. Daniel, K. Sumangala, and C. A. Jeyasehar, "Computer aided modeling and diaphragm design approach for high sensitivity silicon-on-insulator pressure sensors," Measurement, 2011, 44(10): 1924-1936.

[7] S. Li, T. Liang, W. Wang, Y. Hong, T. Zheng, and J. Xiong, "A novel SOI pressure sensor for high temperature application," Journal of Semiconductors, 2015, 36(1): 014014-1-14014-5.

[8] T. H. Lee, S. Bhunia, and M. Mehregany, "Electromechanical computing at $500^{\circ} \mathrm{C}$ with silicon carbide," Science, 2010, 329(5997): 1316-1318.

[9] D. A. Mills, D. Alexander, G. Subhash, and M. Spheplak, "Development of a sapphire optical pressure sensor for high-temperature applications," SPIE, 2014, 9113: 91130H-1-91130H-15.

[10] J. Xu, G. Pickrell, X. Wang, W. Peng, K. Cooper, and A. Wang, "A novel temperature-insensitive optical fiber pressure sensor for harsh environments," IEEE Photonics Technology Letters, 2005, 17(4): 870-872.

[11] C. M. Jewart, Q. Wang, J. Canning, D. Grobnic, S. J. Mihailov, and K. P. Chen, "Ultrafast femtosecond-laser-induced fiber Bragg gratings in air-hole microstructured fibers for high-temperature pressure sensing," Optics Letters, 2010, 35(9): 1443-1445.

[12] A. Binder, G. Bruckner, N. Schobernig, and D. Schmitt, "Wireless surface acoustic wave pressure and temperature sensor with unique identification based on LiNbO3," IEEE Sensors Journal, 2013, 13(5): 1801-1805.

[13] J. G. Rodriguez-Madrid, G. F. Iriarte, O. A. Williams, and F. Calle, "High precision pressure sensors based on SAW devices in the GHz range," Sensors and Actuators A: Physical, 2013, 189(2): 364-369.

[14] K. G. Ong, C. A. Grimes, C. L. Robbins, and R. S. Singh, "Design and application of a wireless, passive, resonant-circuit environmental monitoring sensor," Sensors and Actuators A: Physical, 2001, 93(1): 33-43.

[15] Q. Tan, C. Li, J. Xiong, P. Jia, W. Zhang, J. Liu, et al., "A high temperature capacitive pressure sensor based on alumina ceramic for in situ measurement at $600^{\circ} \mathrm{C}$," Sensors, 2014, 14(2): 2417-2430.

[16] J. Xiong, C. Li, P. Jia, X. Chen, W. Zhang, J. Liu, et al., "An insertable passive LC pressure sensor based on an alumina ceramic for in situ pressure sensing in high-temperature environments," Sensors, 2015, 15(9): 21844-21856. 